\title{
Mathematical Models of the Transient Heat Flow to Fuel Droplets
}

\author{
Herbert S. Bennett and Richard Kayser, Jr. \\ Institute for Materials Research, National Bureau of Standards, Washington, D.C. 20234 \\ (August 12, 1977)
}

\begin{abstract}
Two models for the preheat stage of conventional liquid fuel droplets and of emulsified fuel droplets in combustion gases are analyzed theoretically. These models contain the effects of transient heat conduction to the droplets. In the first model, the droplet and gas temperatures vary temporally but only the gas temperature varies spatially; i.e., the droplet temperature is spatially uniform. Numerical examples, computed from this model, for both the droplet and gas temperatures are given. In the second model, both the droplet and gas temperatures vary spatially and temporally. Numerical examples computed from this second model for the surface and average temperature of the droplet are given. These analyses show that the temperature gradients inside droplets of oil and water are small compared to those in the combustion gases near the droplet; that temperature profiles given by both models are very similar. In particular, the predicted times at which micro-explosions are expected to occur agree within 10 percent of each other.
\end{abstract}

Key words: Combustion; emulsified fuel droplet; fuel droplet; heat flow; micro-explosion; preheat.

\section{Introduction}

Before liquid fuels can burn effectively in combustion chambers, they must be reduced to minute droplets. This atomization of liquid fuels may involve one or more of the following procedures: (1) mixing the fuel with high pressure air or gas; (2) injecting the fuel through small orifices at high pressures; and (3) more recently, superheating emulsified liquid fuels to their "micro-explosive" limit. Effective atomization occurs as follows. First, thin sheets of liquid fuel form. They then become unstable and form ligaments and large drops. Finally, the segments of ligaments and large drops disintegrate further to small droplets [1]. ${ }^{1}$

The ignition of the resulting droplet of conventional fuel consists essentially of two stages [2]. During the first preheat stage, heat flows from the hot surrounding gas to the droplet without significant evaporation. In the second stage, ignition occurs in the gaseous mixture surrounding the droplet and consisting of hot air (oxidizing atmosphere) and fuel vapors. The preheat stage is dominated by transient heat flow and is the subject of this paper.

Several researchers have proposed that emulsified fuels may yield increased combustion efficiencies; decreased pollutant emissions, such as soot, CO, and oxides of nitrogen; and lower maintenance costs [3, 4]. Emulsified fuels consist of two or more components. At least one component has a much higher vapor characteristic than the primary fuel component. The component with the higher vapor pressure, for example water, is dispersed throughout the primary fuel. Such emulsified fuels may be generated by ultrasonic techniques.

The corresponding preheat stage for emulsified fuels consists of two phases. For illustrative purposes, we consider in this paper a two component emulsified fuel. Injection of such an emulsified fuel into a combustor creates compound drops in which the primary fuel surrounds a number of smaller droplets of the dispersed component. Because the smaller droplets have a lower boiling point than that of the surrounding primary fuel, vaporization of the former occurs at superheat temperatures above the normal boiling point. Superheated boiling or spontaneous nucleation of the dispersed component is explosive and occurs at surprisingly reproducible superheat temperatures for ultraclean emulsions $[5,6]$. The resulting sudden change in the volume occupied by the dispersed phase produces catastrophic shattering of the primary fuel drop into a number of much smaller droplets (fragments). This then is the first phase of preheating. The second phase of preheating for emulsified fuels occurs when additional heat transfers to these smaller droplet fragments without significant evaporation and before ignition occurs.

${ }^{1}$ Figures in brackets indicate the literature references at the end of this paper. 
In this paper, we develop two models for predicting the transient heat flow to conventional fuel droplets undergoing preheat and to emulsified droplets undergoing the first phase of preheat. We shall use these models to compute the dependence of the temperature of the droplet and its surrounding, hot oxidizing gas (e.g., air) upon distance from the droplet center and time from injection into the hot oxidizing gas.

In the first model, the droplet and gas temperatures vary temporally but only the gas temperature varies spatially. That is, the droplet temperature is spatially uniform. We shall refer to this model as the zerogradient model. Some researchers propose that in many situations the heat transfer rate within the droplet is much faster than is possible with conductive transfer alone and that internal circulation may be sufficient to maintain a spatially uniform temperature within the droplet [7]. Hence, the zero-gradient model of this paper is an appropriate one for such cases. In the second model, both the droplet and gas temperatures vary spatially and temporally. We shall refer to this second model as the finite-gradient model.

We include these two models for the following reasons. First, the numerical procedures to evaluate the temperature profiles predicted by the zero-gradient model require an order of magnitude less computer time than the numerical procedures to evaluate the temperature profiles predicted by the finite-gradient model require. We expect that the zero-gradient model would be a particularly useful one for any future theoretical analysis of the ignition stage of the droplet. Second, the zero-gradient model readily gives the spatial dependence of the gas temperature near the droplet. We anticipate that such spatial details will be required if one were to consider dense sprays of individual burning particles. And third, we expect that the finitegradient model is the more appropriate one for very short times prior to the micro-explosions of emulsified droplets and for those situations in which the thermal conductivity of the gas is comparable to that of the droplet.

In the next section, we describe the physical assumptions for the preheat stage which are common to both models. Section 3 contains the mathematical description of these assumptions. We derive in section 4 profiles of the droplet and gas which are predicted by the zero-gradient model. In section 5, we give the expressions for the surface temperature of the droplet and for the spatially averaged temperature within the droplet for the finite gradient model. We present in the last section numerical examples for oil-water droplets. We find that the reduced temperatures given by the two models are within 15 percent of each other for small reduced times and are within 5 percent of each other for large reduced times.

\section{Preheat Stage}

In models for droplet preheating, many researchers consider the droplet to be a sphere with temperature independent thermal properties and neglect diffusion effects [2]. Others include thermal diffusion effects but later in their theoretical analyses neglect some terms in the diffusion equations [8]. In addition, most theoretical investigators neglect the influence of gravity, forced convection, and radiant heat transfer. Experimental techniques such as freely falling combustion chambers can be used to eliminate gravitational effects and forced convection. Proper selection of experimental parameters such as ambient pressure and initial droplet size may minimize the other effects not included in the model for preheating. Several researchers have shown that except for heavy fuel oils, radiant heat transfer from the hot gas or from adjacent droplets is negligible [7, 9].

The models examined in this paper contain thermal diffusion effects. We assume that a spherical liquid fuel droplet of radius $r_{\boldsymbol{d}}$ is inserted at time $t=0$ into a hot atmosphere (e.g., air). The initial temperature of the droplet at time $t=0$ is $T_{d 0}$. The hot oxidizing gas is unbounded and at a constant temperature $T_{g 0}$ infinitely far from the droplet. During the preheat stage, we assume that the radius of the droplet does not change. That is, no evaporation occurs. Evaporation will be considered in a subsequent paper.

A conventional fuel droplet is essentially homogeneous and has well defined thermal properties. However, an emulsified fuel droplet composed for example of water and oil, is not microscopically homogeneous. In order to make the models for emulsified droplets amenable to calculation, we must make an additional assertion. For the purposes of transient heat flow analysis, we assert that representative values of the thermal properties of an emulsified fuel droplet exist and that they characterize properly the overall thermal behavior of the droplet. These representative values are essentially uniform throughout the droplet and are most likely bounded by the thermal properties of the fluid components in the emulsified droplet. 
We denote the density, specific heat at constant volume, and thermal conductivity of the droplet by $d_{d}$, $C_{d}$, and $K_{d}$, respectively and of the hot gas by $d_{g}, C_{g}$, and $K_{g}$, respectively. We assume that these thermal properties remain spatially and temporally constant during preheat. In addition, the ambient pressure of the host gas, $p_{g}$, does not change.

Using the above models we seek to compute the dependence of the temperature $T(r, t)$ upon the radial distance $r$ and time $t$. In particular, we shall compute the time required for a conventional fuel droplet to reach a given temperature and for the emulsified fuel droplet to reach its superheat limit $T_{\mathrm{SL}}$; i.e., the temperature near which the micro-explosion occurs. Because the adsorption of impurities at the interface between liquids might alter surface and interfacial tension, most water droplets in oil vaporize explosively somewhere between 240 and $270^{\circ} \mathrm{C}[3,5]$.

\section{Theoretical Analysis}

We find it convenient at this point to introduce several dimensionless quantities. We denote the reduced temperature $\theta(\eta, \tau)$ by the expression

$$
\left.\theta(\eta, \tau)=\left[T(r, t)-T_{d 0}\right) /\left(T_{g 0}-T_{d 0}\right)\right],
$$

The dimensionless distance is $\eta=\left(r / r_{d}\right)$ and the dimensionless time is $\tau=\left(t / t_{d}\right)$. In this paper, we shall denote dimensionless quantities by Greek letters. The time $t_{d}$ is proportional to the time required for a heat pulse to diffuse across the droplet and is given by $t_{d}=r_{d}{ }^{2} / a_{d}{ }^{2}$ where $a_{d}{ }^{2}=\left(K_{d} / d_{d} C_{d}\right)$ denotes the thermal diffusivity of the droplet. Even though the reduced time $\tau$ may be mathematically unbounded, it does have a physically determined upper limit for the preheat stage. The physically meaningful values of $\tau$ must be less than that value of $\tau=\tau_{\max }$ for which $\theta\left(\eta, \tau_{\max }\right)$ corresponds to the boiling point of the liquid in the droplet.

The Fourier heat conduction equations for the droplet and its surrounding gas describe the time and space dependence of the temperature when local thermodynamic equilibrium exists. For the case in which the isothermal surfaces are concentric spheres centered about the droplet center at $\eta=0$, these equations are, for $\tau \geq 0$ and $\eta<1$ (inside the droplet)

$$
\frac{\partial^{2} \theta_{d}}{\partial \eta^{2}}+\frac{2}{\eta} \frac{\partial \theta_{d}}{\partial \eta}=\frac{\partial \theta_{d}}{\partial \tau}
$$

and for $\tau \geq 0$ and $\eta>1$ (outside the droplet)

$$
\frac{\partial^{2} \theta_{g}}{\partial \eta^{2}}+\frac{2}{\eta} \frac{\partial \theta_{g}}{\partial \eta}=\alpha^{2} \frac{\partial \theta_{g}}{\partial \tau}
$$

where $\eta \geq 0$ and $\alpha=a_{d} / a_{g}$. The quantity $a_{g}{ }^{2}=\left(K_{g} / d_{g} C_{g}\right)$ is the thermal diffusivity of the gas and the subscripts $d$ and $g$ refer respectively to the droplet and the gas.

These Fourier heat conduction equations require a statement of the boundary conditions before solutions are uniquely defined. The reduced temperature $\boldsymbol{\theta}$ has the form

$$
\theta_{d}(\eta, \tau)=0 \text { for } \eta<1 \text { and } \tau \leq 0
$$

and

$$
\theta_{g}(\eta, \tau)=1 \quad \text { for } \quad \eta>1 \text { and } \tau \leq 0
$$

and it becomes for $\boldsymbol{\tau}>0$

$$
\theta(\eta, \tau)= \begin{cases}\theta_{d}(\eta, \tau) & \text { for } \quad \eta<1 \\ \theta_{g}(\eta, \tau) & \text { for } \quad \eta>1\end{cases}
$$


The boundary conditions do not depend explicitly upon time. The temperature is finite everywhere namely, $\theta_{d}(0, \tau)$ is finite and

$$
\operatorname{Lim}_{\eta \rightarrow \infty} \theta_{g}(\eta, \tau)=1 .
$$

Also, the condition that no heat flux exists at the center of the droplet becomes

$$
\left.\frac{\partial \theta_{d}(\eta, \tau)}{\partial \eta}\right|_{\eta=0}=0
$$

The continuity of the temperature across the droplet-gas interface is

$$
\theta_{d}(1, \tau)=\theta_{g}(1, \tau) .
$$

The conservation of heat flow at the interface gives,

$$
\left.\frac{\partial \theta_{d}(\eta, \tau)}{\partial \eta}\right|_{\eta=1}=\left.\beta \frac{\partial \theta_{g}(\eta, \tau)}{\partial \eta}\right|_{\eta=1},
$$

where $\beta=K_{g} / K_{d}$ is the ratio of thermal conductivities for the gas and droplet.

Equations (2) through (7) represent the mathematical description of the above model for transient heat flow during the preheat stage of an individual fuel droplet injected at time $t=0$ into hot oxidizing gases. We solve the equations by taking their Laplace transforms with respect to time. The Laplace transform of the reduced temperature $\boldsymbol{\theta}(\boldsymbol{\eta}, \tau)$ is denoted by

$$
\Phi(\eta, \sigma)=\int_{0}^{\infty} \exp (-\sigma \tau) \theta(\eta, \tau) d \tau,
$$

where $\sigma$ is the dimensionless Laplace transform variable. The Bromwich integral

$$
\theta(\eta, \tau)=\operatorname{Lim}_{\delta \rightarrow \infty} \frac{1}{2 \pi i} \int_{\epsilon-i \delta}^{\epsilon+i \delta} \exp (\sigma \tau) \Phi(\eta, \sigma) d \sigma,
$$

expresses the temperature in terms of the Laplace transform. The quantity $\epsilon$ is chosen sufficiently large so that the integral,

$$
\int_{0}^{\infty} \exp (-\sigma \tau)|\theta(\eta, \tau)| d \tau
$$

exists.

Introducing the dimensionless variable $X=\sigma^{1 / 2} \eta$, we express the Laplace transforms of eqs (2) through (7), respectively in the forms

$$
\begin{gathered}
\left\{\frac{\partial^{2}}{\partial \chi^{2}}+\frac{2}{\chi} \frac{\partial}{\partial \chi}-1\right\} \Phi_{d}(\chi)=0 \\
\left\{\frac{\partial^{2}}{\partial^{2}}+\frac{2}{\chi} \frac{\partial}{\partial \chi}-\alpha^{2}\right\} \Phi_{g}(\chi)=-\frac{\alpha^{2}}{\sigma} \\
\left.\frac{\partial \Phi_{d}}{\partial \chi}\right|_{\chi=0}=0
\end{gathered}
$$




$$
\begin{gathered}
i m_{\eta \rightarrow \infty} \Phi_{g}(\chi)=\sigma^{-1} \\
\Phi_{d}(\chi=\rho)=\Phi_{g}(\chi=\rho) \\
\left.\frac{\partial \Phi_{d}}{\partial}\right|_{\chi=\rho}=\left.\beta \frac{\partial \Phi_{g}}{\partial \chi}\right|_{\chi=\rho},
\end{gathered}
$$

where $\rho=\sigma^{1 / 2}$.

The solution to eq (9) and the condition (11) require that the Laplace transform for the droplet has the form

$$
\Phi_{d}(\chi)=\gamma_{d} \chi^{-1} \sinh (\chi)
$$

Similarly, with $\chi_{g}=\alpha \chi$, the solution to eq (10) and the condition (12) require that

$$
\Phi_{g}\left(\chi_{g}\right)=\gamma_{g} \chi_{g}{ }^{-1} \exp \left(\chi_{g}\right)+\rho^{-2} .
$$

The remaining continuity condition (13) and the conservation of heat flow eq (14) yield two simultaneous, inhomogeneous equations for the coefficients $\gamma_{d}$ and $\gamma_{g}$. Solving these equations, we obtain the respective Laplace transforms for the droplet and the gas;

$$
\Phi_{d}(\chi)=\Delta^{-1} \beta(1+\alpha \rho) \sinh (\chi) \quad \text { for } \quad \eta<1
$$

and

$$
\Phi_{g}(\chi)=-\Delta^{-1}\{\rho \cosh (\rho)-\sinh (\rho)\} \exp \{-\alpha \rho(\eta-1)\}+\rho^{-2},
$$

for $\eta>1$, and where

$$
\Delta=\rho^{2} \eta[\{\beta(1+\alpha \rho)-1\} \sinh (\rho)+\rho \cosh (\rho)] .
$$

Inserting eqs (17) and (18) respectively into the Bromwich integral (8) gives us the reduced temperatures $\theta_{d}(\eta, \tau)$ for the droplet and $\theta_{g}(\eta, \tau)$ for the gas. In the following section 4 , we give expressions predicted by the zero gradient model for the reduced temperature $\boldsymbol{\theta}_{\boldsymbol{d}}$ in the droplet and for the reduced temperature $\boldsymbol{\theta}_{\boldsymbol{g}}$ in the gas near the droplet. In section 5 , we present the exact evaluation of the reduced temperature $\boldsymbol{\theta}_{\boldsymbol{d}}$ inside and on the surface of the droplet for the finite gradient model.

\section{Zero-Gradient Model}

Two different but equivalent ways exist for obtaining the expressions which correspond to the zerogradient model. We shall give both.

First, for sufficiently large reduced times $\tau$, the major contributions to the Bromwich integral (8) arise from the region corresponding to values of $|\boldsymbol{\sigma}|=\left|\rho^{2}\right|$ which are less than one. This suggests that we consider expansions in powers of $\boldsymbol{\rho}$. We divide eq (17) and (19) by sinh $(\boldsymbol{\rho})$ and for small values of $\rho$ approximate $\rho$ $\cosh (\rho)$ by $1+\left(\rho^{2} / 3\right)$ and $\sinh (\rho)$ by $\rho$. For even smaller values of $\chi=\eta \rho$ because $\eta \leq 1$, we approximate $\sinh (\chi)$ by $\chi$ and find that,

$$
\Phi_{d}(\chi) \rightarrow \Phi_{d z}(\chi)=3 \beta(1+\alpha \rho)\left[\rho^{2}\left(\rho+\rho_{+}\right)\left(\rho+\rho_{-}\right)\right]^{-1},
$$

where

$$
\rho_{ \pm}=(3 \alpha \beta / 2)\left[1 \pm\left\{1-\left(4 / 3 \beta \alpha^{2}\right)\right\}^{1 / 2}\right] .
$$

We apply the partial fraction method to eq (24) and rewrite it in the form,

$$
\Phi_{d z}(\chi)=\rho^{-2}-\left[\left(\rho+\rho_{+}\right)\left(\rho+\rho_{-}\right)\right]^{-1} .
$$


Observe that for sufficiently long reduced times $\tau$, the droplet reduced temperature $\theta_{\boldsymbol{d}}$ becomes independent of $\eta$; i.e., it is spatially uniform and depends only upon the reduced time $\tau$.

Procedures similar to that by which we obtain eq $(20)$ give us that

$$
\Phi_{g}(\chi) \rightarrow \Phi_{g z}(\chi)=\rho^{-2}-\left[\eta\left(\rho+\rho_{+}\right)\left(\rho+\rho_{-}\right)\right]^{-1} \exp \{-\alpha \rho(\eta-1)\} .
$$

Using the relation that

$$
\left[\left(\rho+\rho_{+}\right)\left(\rho+\rho_{-}\right)\right]^{-1}=\left(\rho_{-}-\rho_{+}\right)^{-1}\left\{\left(\rho+\rho_{+}\right)^{-1}-\left(\rho+\rho_{-}\right)^{-1}\right\}
$$

and referring to page 1023 and page 1026 of Abramowitz and Stegun [10], for $\boldsymbol{\eta}<1$, we evaluate the Bromwich integrals for eqs (22) and (23). The results are, for $\boldsymbol{\eta}<1$,

$$
\begin{aligned}
\theta_{d z}(\tau)=1+ & \left(\rho_{+}-\rho_{-}\right)^{-1}\left\{-\rho_{+} \exp \left(\rho_{+}{ }^{2} \tau\right) \operatorname{erfc}\left(\rho_{+} \sqrt{\tau}\right)\right. \\
& \left.+\rho_{-} \exp \left(\rho_{-}{ }^{2} \tau\right) \operatorname{erfc}\left(\rho_{-} \sqrt{\tau}\right)\right\}
\end{aligned}
$$

and for $\boldsymbol{\eta}>1$,

$$
\begin{aligned}
& \boldsymbol{\theta}_{g z}(\eta, \tau)=1+\left\{\eta\left(\rho_{+}-\rho_{-}\right)\right\}^{-1}\left[-\rho_{+} \exp \left\{\boldsymbol{\rho}_{+}{ }^{2} \tau+\alpha \rho_{+}(\eta-1)\right\}\right. \\
& \operatorname{erfc}\left\{\rho_{+} \sqrt{ } \boldsymbol{\tau}+\right.(\alpha(\eta-1) / 2 \sqrt{\tau})\}+\rho_{-} \exp \left\{\boldsymbol{\rho}_{-}{ }^{2} \tau+\alpha \rho(\eta-1)\right\} \\
& \operatorname{erfc}\left\{\boldsymbol{\rho}_{-} \sqrt{\boldsymbol{\tau}}+(\alpha(\eta-1) / 2 \sqrt{\tau})\right]
\end{aligned}
$$

where the subscripts $d z$ and $g z$ denote the droplet and gas reduced temperatures for the zero-gradient model and where the complementary error function of complex argument $z$ is denoted by erfc $(z)$. Equations (25) and (26) are valid only when $\rho_{+} \neq \rho_{-}$.

For fuel droplets in combustion gases, the values of $\alpha$ and $\beta$ are such that $\rho_{+}$equals the complex conjugate of $\rho_{-}$; i.e., $\rho_{+}=\rho_{-} *$. We then analytically continue the functions appearing in eqs (25) and (26) to their values for complex arguments in order to compute the reduced temperatures. This is a tedious process and we refer the reader to [11] for the details.

In order to compute the reduced times $\tau$ for which eqs (25) and (26) correspond to valid inverse transforms of eqs (17) and (18), we consider the previous inequality $\sigma^{1 / 2}<1$. We divide both sides of this inequality by $\sigma$ and then take the inverse Laplace transform. This yields the inequality $(\pi \tau)^{-1 / 2}<1$ or $\tau>\pi^{-1}$. Remembering that $\tau=\left(t / t_{d}\right)$, we have that for times $t>\left(r_{d}{ }^{2} / \pi a_{d}{ }^{2}\right)$ the temperature gradients in the droplet become negligible. The time $\left(r_{\boldsymbol{d}}{ }^{2} / \boldsymbol{\pi}_{\boldsymbol{d}}{ }^{2}\right)$ corresponds to the time which is required for a heat pulse to diffuse a distance $r_{d}$ in the droplet.

There is a second way in which to obtain the same reduced temperatures given by eqs (25) and (26) without making any assertions about the value of $\tau$. The second argument involves considering the conservation of heat flow at the interface for the case in which the total thermal conductivity of the droplet due to both Fourier heat conduction and internal circulation exceeds that for the gas. As we mentioned in section 1, the internal circulation inside a droplet may be sufficient in many cases to maintain a spatially uniform temperature within the droplet. When such conditions prevail, the Fourier heat conduction equation for the droplet, eq (2), and the boundary condition (7), are replaced by a single heat transfer equation. For the case in which the droplet temperature is spatially uniform, $T_{d}(r, t)=T_{d}(t)=T_{g}\left(r_{d}, t\right)$, the heat flux into the droplet,

$$
\left\{\left(4 \pi r_{d}^{3} / 3\right) d_{d} C_{d}\left(d T_{d} / d t\right) / 4 \pi r_{d}^{2}\right\}
$$

must equal the heat flux from the gas, $K_{g}\left(\partial T_{g}(r, t) / \partial r\right)_{r=r_{d}}$. This last equality replaces eqs (2) and (7) and becomes in terms of the dimensionless quantities used in this paper,

$$
\frac{1}{3} \frac{d \theta_{d v}}{d \tau}=\left.\beta \frac{\partial}{\partial \eta} \theta_{g u}(\eta, \tau)\right|_{\eta=1}
$$


where $\theta_{d v}$ and $\theta_{g u}$ are the reduced temperatures for the spatially uniform case discussed above. The Laplace transform of eq (28) is

$$
\frac{\sigma}{3} \Phi_{d u}(\sigma)=\left.\beta \frac{\partial}{\partial \eta} \theta_{g u}(\eta, \sigma)\right|_{\eta=1}
$$

and this eq (29) replaces eqs (9) and (14). Remembering that $\Phi_{d u}(\sigma)=\Phi_{g u}(1, \sigma)$, we use eqs (10) and (12) with $\Phi_{g}$ replaced by $\Phi_{g u}$ and eq (29) to obtain the result that $\Phi_{g u}(\eta, \sigma) \equiv \Phi_{g z}(\chi)$, where $\Phi_{g z}(\chi)$ is given by eq (23). Hence, we then have that for all values of reduced time $\tau \geq 0$ and when $\left(\partial T_{d}(r, t) / \partial r\right)=0$ for all $r$ $\leq r_{0}$, the reduced droplet temperature is $\boldsymbol{\theta}_{d u}(\tau)=\boldsymbol{\theta}_{d z}(\tau)$ and $\boldsymbol{\theta}_{g u}(\boldsymbol{\eta}, \boldsymbol{\tau})=\boldsymbol{\theta}_{\boldsymbol{g} z}(\boldsymbol{\eta}, \boldsymbol{\tau})$, where $\boldsymbol{\theta}_{\boldsymbol{d z}}(\tau)$ and $\boldsymbol{\theta}_{\boldsymbol{g} z}(\boldsymbol{\eta}$, $\tau)$ are given by eqs (25) and (26).

For the case of an oil or water droplet in hot air at atmospheric pressure, the quantity $\left(4 / 3 \beta \alpha^{2}\right)$ is of the order of $10^{4}$ and the quantities $\rho_{+}$and $\rho_{-}$are approximated very well by $\rho_{ \pm} \approx \pm i(3 \beta)^{1 / 2}$. Using these values in eqs. (22) and (23) and assuming that $\alpha(\eta-1) \ll 1$ in eq (23), we obtain the rather simple approximations to eq (25) and to eq (26); namely,

$$
\operatorname{Lim}_{\alpha \rightarrow 0} \theta_{d z}(\tau)=\theta_{d 0}(\tau)
$$

and

$$
\operatorname{Lim}_{\alpha \rightarrow 0} \theta_{g z}(\eta, \tau)=\theta_{g 0}(\eta, \tau),
$$

where

$$
\theta_{d 0}(\tau)=1-\exp (-3 \beta \tau)
$$

and

$$
\theta_{g o}(\eta, \tau)=1-\eta^{-1} \exp (-3 \beta \tau) .
$$

From eq $(30)$, we observe that $(3 \beta)^{-1}$ is the reduced time required for $\left(1-\boldsymbol{\theta}_{\boldsymbol{d} 0}\right)$ to reach $\left(\boldsymbol{e}^{-\mathbf{1}}\right)$ times its value at $\tau=0$. We shall see in section 6 that for oil and water droplets in air, the numerical predictions of eqs (30) and (31) agree very well with the numerical predictions of the exact equations (25) and (26).

\section{Finite Gradient Model}

In the finite gradient model, the reduced temperature distributions of the droplet and the surrounding gas are obtained by directly evaluating the Bromwich integrals,

$$
\theta(\eta, \tau)=\operatorname{Lim}_{\delta \rightarrow \infty} \frac{1}{2 \pi i} \int_{\epsilon-i \delta}^{\epsilon+i \delta} \exp (\sigma \tau) \Phi(\eta, \sigma) d \sigma .
$$

$\Phi(\eta, \sigma)$, or equivalently $\Phi(\chi)$ with $\chi=\sigma^{1 / 2} \eta$, is given by eq (17) for the droplet and eq (18) for the surrounding gas.

To evaluate the Bromwich integral it is first necessary to investigate the nature of the integrand exp $(\sigma \tau) \Phi(\eta, \sigma)$. No distinction will be made in this discussion between the droplet and the gas as the integrands in both cases exhibit the same general properties. Since $\exp (\sigma \tau) \Phi(\eta, \sigma)$ is not an even function of $\chi$ it is not a single valued function of $\sigma$, and it possesses a branch-point at $\sigma=0$. The quantity $\exp$ $(\sigma \theta) \Phi(\eta, \sigma)$ also has a simple first-order pole with residue unity at $\sigma=0$ and singularities at the nonzero roots of $\Delta$ given in eq (19). Simple considerations of $\Delta$ indicate that no pure real or pure imaginary roots exist; but they do not rule out the existence of complex roots. We present in appendix A a general proof that the function $\Delta$ does not have any complex roots [12]. 
We now evaluate the Bromwich integral. We compute the contour of equation (32) by two quarter circular arcs in the upper and lower left hand plane, by two parallel lines running above and below the negative real $\sigma$-axis, and by a small circular arc traversing a path around the origin $\sigma=0$. The reduced temperature distributions for the droplet and the gas are then obtained by applying Caueky's Theorem and by letting $\delta \rightarrow$ $\infty$ in eq (8) and the radius of the smaller circle about the origin tend to zero. The quantity $\epsilon$ is taken to be an arbitrarily small positive number. The integrations along the quarter-circular arcs vanish as their radii go to infinity. The simple first order pole at $\sigma=0$ contributes unity, and the two infinite integrals above and below the negative real $\sigma$-axis are evaluated with $\sigma$ set equal to $\xi^{2} i^{i \pi}$ and $\xi^{2} e^{-i \pi}$, respectively. We then obtain the following expressions for the reduced temperature distributions:

$$
\theta_{d}(\eta, \tau)=1+\frac{2 \alpha \beta}{\pi \eta} \int_{0}^{\infty} \frac{\sin \xi \eta(\xi \cos \xi-\sin \xi) \exp \left(-\tau \xi^{2}\right) d \xi}{\alpha^{2} \beta^{2} \xi^{2} \sin ^{2} \xi+(\xi \cos \xi-(1-\beta) \sin \xi)^{2}}
$$

and

$$
\theta_{g}(\eta, \tau)=1+\frac{2}{\pi \eta} \int_{0}^{\infty} \frac{N(\eta, \xi)(\xi \cos \xi-\sin \xi) \exp \left(-\tau \xi^{2}\right) d \xi}{\alpha^{2} \beta^{2} \xi^{2} \sin ^{2} \xi+(\xi \cos \xi-(1-\beta) \sin \xi)^{2}}
$$

where $N(\boldsymbol{\eta}, \boldsymbol{\xi})=\boldsymbol{\xi}^{-1} \sin (\alpha(\boldsymbol{\eta}-1) \boldsymbol{\xi})[\boldsymbol{\xi} \cos \boldsymbol{\xi}-(1-\beta) \sin \boldsymbol{\xi}]+\alpha \beta \sin \boldsymbol{\xi} \cos (\boldsymbol{\alpha}(\boldsymbol{\eta}-1) \boldsymbol{\xi})$. The average temperature of the droplet is given by

$$
\begin{aligned}
\theta_{d}(\operatorname{avg}, \tau) & =\int_{0}^{1} \eta^{2} \theta_{d}(\eta, \tau) \mathrm{d} \eta / \int_{0}^{1} \eta^{2} \mathrm{~d} \eta \\
& =1-\frac{6 \alpha \beta}{\pi} \int_{0}^{\infty} \frac{\xi^{-2}(\xi \cos \xi-\sin \xi)^{2} \exp \left(-\tau \xi^{2}\right) d \xi}{\alpha^{2} \beta^{2} \xi^{2} \sin ^{2} \xi+(\xi \cos \xi-(1-\beta) \sin \xi)^{2}},
\end{aligned}
$$

and a similar expression for the surrounding gas may easily be obtained from eq (34).

The above rather complicated integrals must be evaluated numerically. A short outline of the procedure which we used is given in appendix B.

We discuss in the next section the representative values of the reduced surface temperature of the droplet $\boldsymbol{\theta}_{\boldsymbol{g}}(\tau) \equiv \boldsymbol{\theta}_{\boldsymbol{g}}(1, \tau)$ given by eq (33) with $\boldsymbol{\eta}=1$ and the spatially averaged reduced temperature throughout the droplet $\theta_{\mathrm{av}}(\tau) \equiv \theta_{d}(\mathrm{avg}, \tau)$ given by eq (35).

\section{Numerical examples and Conclusions}

In this section, we give some illustrative numerical examples for the predictions made by the zero-gradient and finite gradient models. The input data for these calculations are the thermal conductivity, the density, and the specific heat of the fuel droplet and the combustion gas. Values for the above properties of a conventional fuel droplet are readily available. Table 1 contains typical values of medium weight fuel oils.

TABLE 1. Thermal properties

The thermal conductivity, the density, and the specific heat at constant volume are denoted respectively by $K, d$, and $C$.

\begin{tabular}{cccc}
\hline \hline & $K$ & $d$ & $C$ \\
\hline \multirow{2}{*}{ oil } & $\left(\mathrm{J} / \mathrm{cm} \mathrm{s} \mathrm{K}^{-3}\right.$ & $\left(\mathrm{g} / \mathrm{cm}^{3}\right)$ & $(\mathrm{J} / \mathrm{g} \mathrm{K})$ \\
water & $6.75 \times 10^{3}$ & 0.95 & 2.24 \\
gas & $2.70 \times 10^{-4}$ & 1.00 & 4.20 \\
\hline
\end{tabular}

Values for the above properties of an emulsified fuel require more consideration. We expect that an emulsified droplet containing oil and water will have thermal properties which are bounded by those for the 
pure fuel oil and pure water components. Hence, we list also in table 1 the values for the thermal properties of water. An emulsified droplet of 10 per cent water and 90 per cent fuel oil probably has an overall thermal behavior which is very close to that of fuel oil. Nonetheless, we include calculations for the pure water droplets to give bounds on the predictions and to test the sensitivity of the predictions upon the thermal properties of the droplet. Past experience indicates that whenever the thermal conductivity of the spherical droplet being heated exceeds the thermal conductivity of the medium surrounding the droplet, the thermal response of the droplet-gas system is dominated substantially by the thermal properties of the surrounding medium. We use the values of air at one atmosphere of pressure to represent the hot oxidizing combustion gases. These values are also given in table 1. Table 2 contains values for the two quantities $\alpha$ and $\beta$. The radius of the droplet $r_{\boldsymbol{d}}$ is $50 \mu \mathrm{m}$ for all the numerical examples considered in this paper.

TABLE 2. Reduced parameters

The dimensionless quantity $\alpha^{2}$ is the thermal diffusivity of the droplet $\alpha_{d}^{2}=\left(K_{d} / d_{a} C_{d}\right)$ divided by the thermal diffusivity of the gas $\alpha_{g}^{2}=\left(K_{g} / d_{g} C_{g}\right)$ and the dimensionless quantity $\beta$ is the thermal conductivity of the gas $K_{g}$ divided by the thermal conductivity of the droplet. The density and specific heat of the droplet and the gas are denoted respectively by $d_{\boldsymbol{d}}$ and $C_{\boldsymbol{d}}$ and by $d_{\boldsymbol{g}}$ and $C_{\boldsymbol{g}}$.

oil droplet

water droplet

\subsection{2}

0.0880

$\beta$

0.186

0.040

Figures 1 and 2 compare respectively the numerical predictions of the zero gradient and finite gradient models for oil droplets in air. Figures 3 and 4 compare respectively the numerical predicitions of the zero gradient and finite gradient models for water droplets in air. From these figures, we observe that the four reduced temperatures $\theta_{d z}(\tau), \theta_{d 0}(\tau), \theta_{s}(\tau)$, and $\theta_{\text {av }}(\tau)$ all agree to within 15 per cent of each other for values of $\tau \lessgtr 0.5$ and to within 5 per cent of each other for values of $\tau \approx 2.5$. Because all four quantities approach unity as $\tau$ approaches infinity, the agreement among the values improves for values of $\tau>2.5$. We also find that the water droplets require longer reduced times $\tau$ to achieve reduced temperatures comparable to those of the oil droplet.

Because the computer time required to evaluate $\theta_{s}(\tau)$ and $\theta_{\text {av }}(\tau)$ exceeds the computer time to evaluate $\theta_{d z}(\tau)$ by at least a factor of ten and because the numerical agreement among $\theta_{d z}(\tau), \theta_{\boldsymbol{g}}(\tau)$, and $\boldsymbol{\theta}_{\mathrm{av}}(\tau)$ is good for oil and water droplets in air whenever $\tau>0.5$, we can envisage situations for which $\boldsymbol{\theta}_{\boldsymbol{d z}}(\tau)$ evaluations will be adequate. From these numerical examples, we conclude then that whenever $\beta<1$ and for $\tau>0.5$, the numerical predictions of the zero-gradient and finite-gradient models quantitatively agree to within 10 per cent. This of course will not be true if $\beta>1$. Hence, for the oil and water droplets considered here, the predictions of the zero-gradient and finite-gradient models differ significantly only for values of $\tau$ substantially less than 0.25 .

Another quantity which enters the design of combustion chambers is the time required to achieve the superheat limit of emulsified fuel droplets; i.e., the time required before the emulsified fuel droplet undergoes a "microexplosion." For illustrative purposes, let us assume that the superheat limit $T_{\mathrm{SL}}$ for an oil-water emulsification is $260{ }^{\circ} \mathrm{C}$, and the droplet temperature $T_{\boldsymbol{d} 0}$ is $20^{\circ} \mathrm{C}$, and the gas temperature $T_{\boldsymbol{g} 0}$ is $1520{ }^{\circ} \mathrm{C}$. The superheat limit that corresponds to the reduced temperature $\theta_{\mathrm{SL}}=0.16$. From the numerical examples, we find that for pure oil droplets, $\theta_{d 0}\left(\tau_{d 0}^{0}=0.313\right)=0.16, \theta_{d z}\left(\tau_{d z}^{0}=0.317\right)=0.16, \theta_{8}\left(\tau_{8}^{0}=\right.$ $0.233)=0.16$, and $\theta_{\mathrm{av}}\left(\tau_{\mathrm{av}}^{0}=0.286\right)=0.16$. The corresponding reduced time values at the superheat limit for pure water droplets are

$$
\begin{aligned}
\theta_{d 0}\left(\tau_{d 0}^{w}=1.45\right) & =0.16, \quad \theta_{d z}\left(\tau_{d z}^{w}(=1.47)=0.16\right. \\
\theta_{s}\left(\begin{array}{c}
w \\
s
\end{array}=1.29\right) & =0.16, \quad \text { and } \quad \theta_{\mathrm{av}}\left(\tau_{a v}^{w}=1.36\right)=0.16
\end{aligned}
$$

Using the values of $t_{\boldsymbol{d}}=36.8 \mathrm{~ms}$ for oil and $t_{\boldsymbol{d}}=15.6 \mathrm{~ms}$ for pure water, we list the superheat times predicted by the two models for pure oil droplets; namely, $t_{d 0}^{0}=11.5 \mathrm{~ms}, t_{d z}^{0}=11.7 \mathrm{~ms}, t_{8}^{0}=8.7 \mathrm{~ms}$ and $t_{a v}^{0}=10.5 \mathrm{~ms}$. The corresponding values for pure water droplets are $t_{d 0}^{2}=22.5 \mathrm{~ms}, t_{d z}^{2}=22.9 \mathrm{~s}, t_{s}^{\nu}=$ $20.1 \mathrm{~ms}$, and $t_{\mathrm{av}}=21.1 \mathrm{~ms}$. Again, the quantitative differences between the two models for the cases 


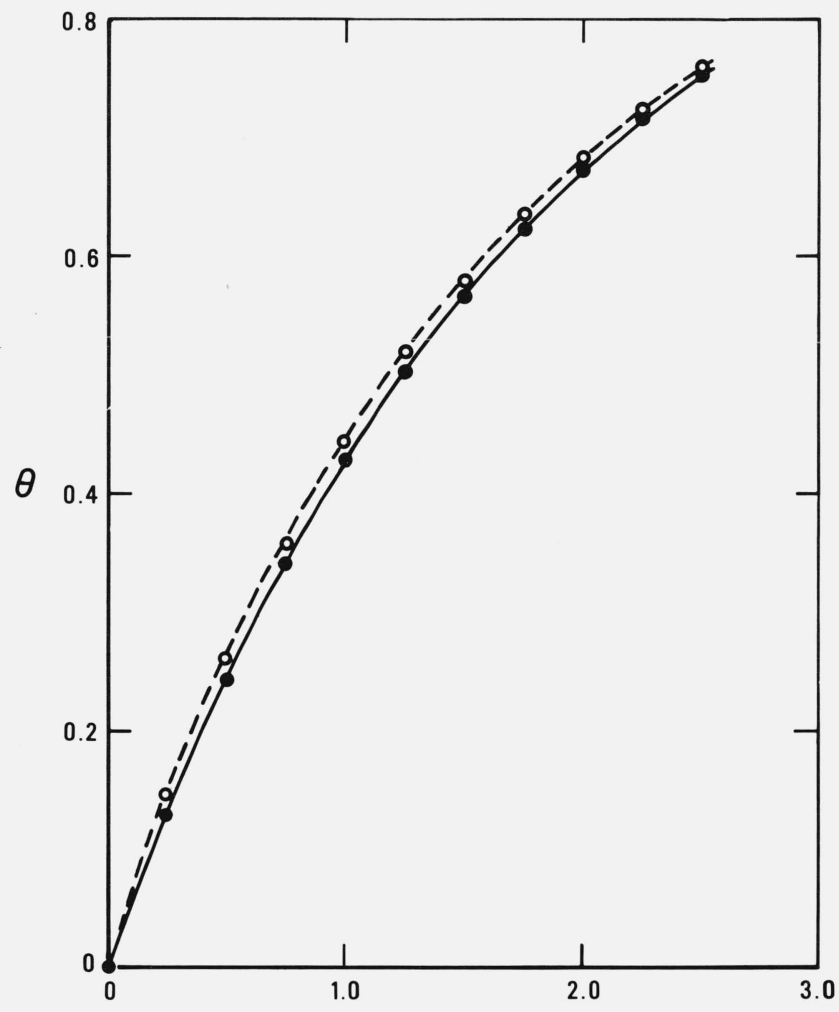

FigURE 1. The reduced temperature $\boldsymbol{\theta}$ as a function of reduced time $\boldsymbol{\tau}$ predicted by the zero gradient model for oil droplets in air.

The solid curve with solid dots corresponds to the reduced temperature $\theta_{0 z}$ given by eq (26). The dashed curve with open circles corresponds to the reduced temperature $\theta_{\text {do }}$ given by eq. (31). All of the above quantities are dimensionless and the droplet radius is $50 \mu \mathrm{m}$.

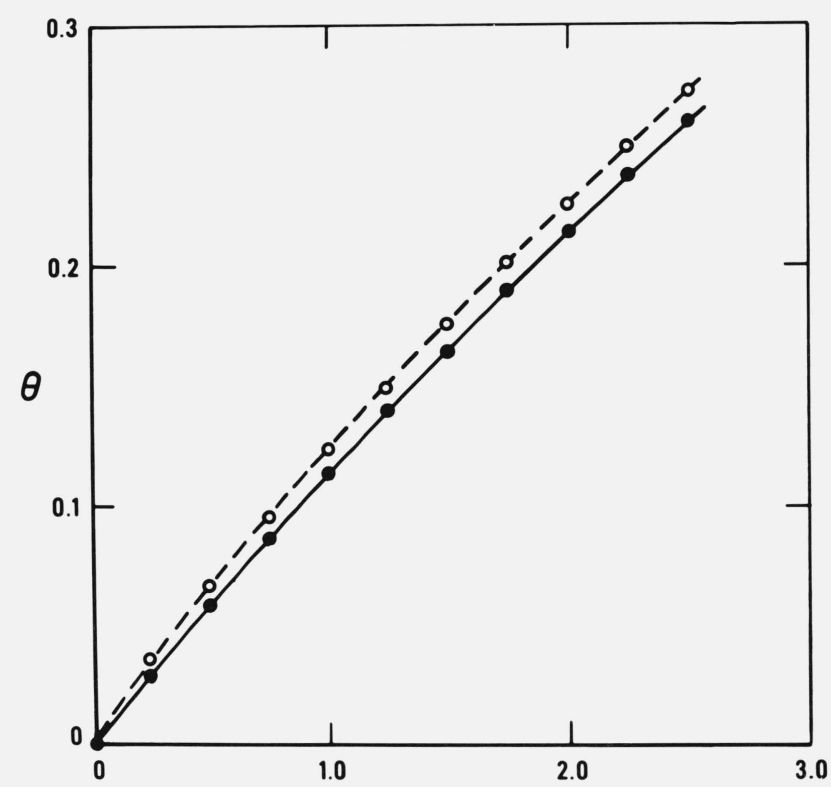

Figure 3. The reduced temperature $\theta$ as a function of reduced time $\tau$ predicted by the zero gradient model for water droplets in air.

The solid curve with solid dots corresponds to the reduced temperature $\theta_{d z}$, eq (26), and the dashed curve with open circles corresponds to the reduced temperature $\boldsymbol{\theta}_{\mathrm{do}}$, eq (31). All of the above quantities are dimensionless and the radius of the droplet is $50 \mu \mathrm{m}$.

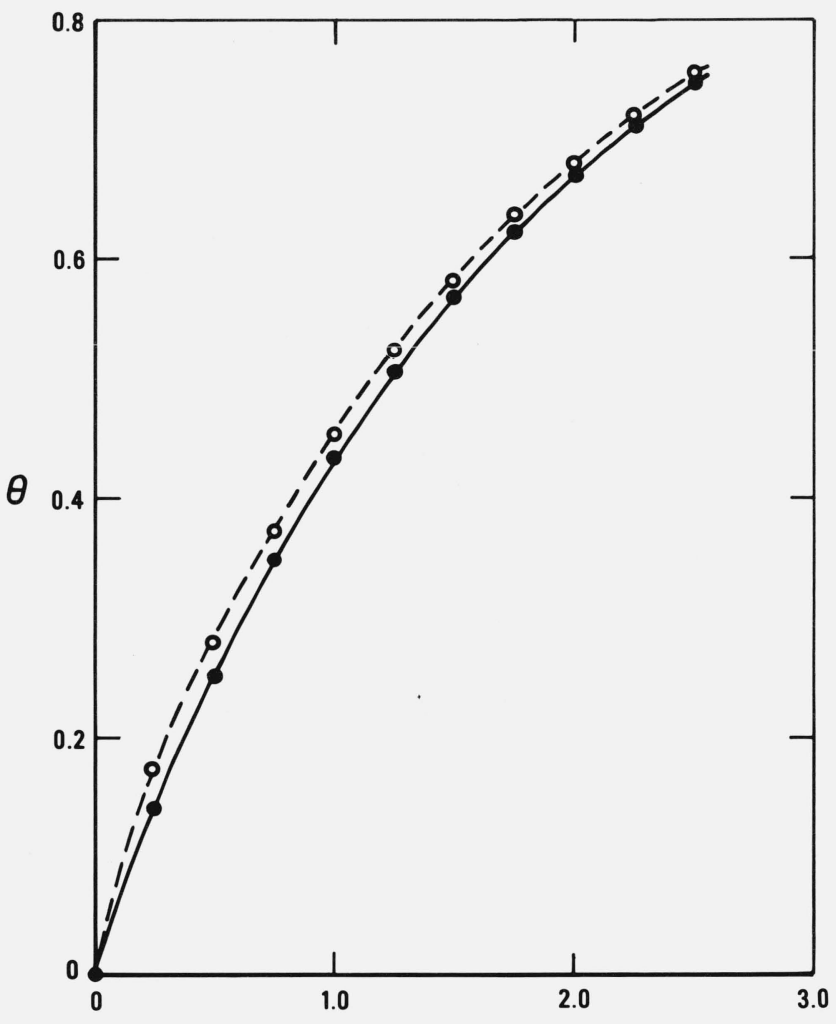

FIGURE 2. The reduced temperature $\boldsymbol{\theta}$ as a function of reduced time $\tau$ predicted by the finite gradient model for oil droplets in air.

The solid curve with solid dots is the average droplet temperature $\theta_{\text {av }}$ given by eq (36) and the dashed curve with open circles is the surface temperature of the droplet $\theta_{s}$ given by eq (34) with $\eta=$ dashed curve with open circles is the surface temperature of the droplet $\theta_{s}$ given by eq
1 . All of the above quantities are dimensionless and the radius of the droplet is $50 \mu \mathrm{m}$.

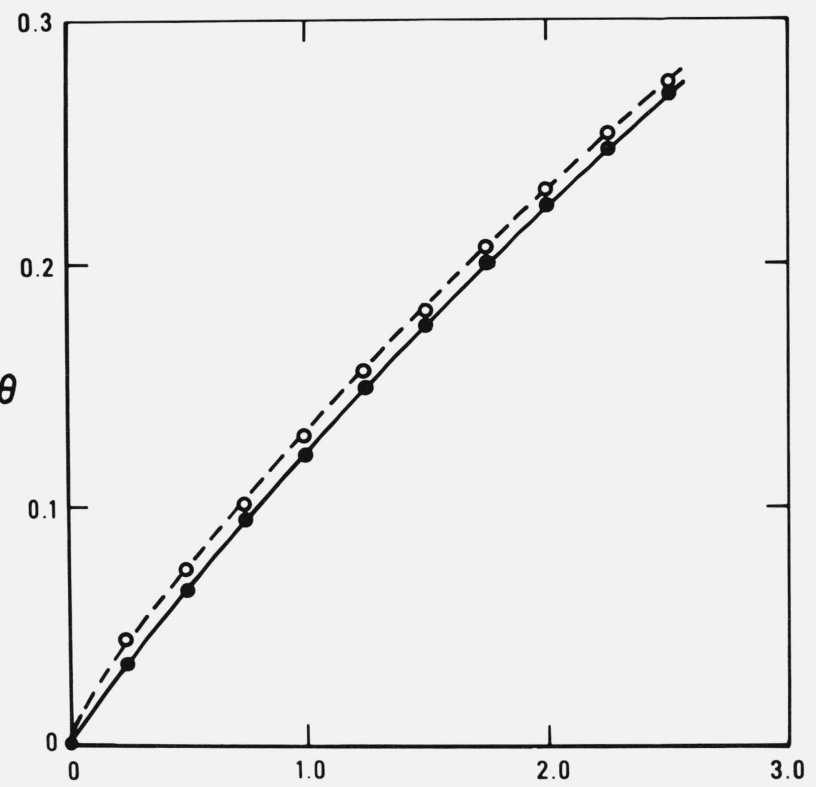

FIGURE 4. The reduced temperature $\theta$ as a function of reduced time $\tau$ predicted by the finite gradient model for water droplets in air.

The solid curve with dots is the average droplet temperature $\theta_{a v}$, eq (36), and the dashed curve with open circles is the surface temperature of the droplet $\theta_{s}$, eq (34), with $\eta=1$. All of the above quantities are dimensionless and the radius of the droplet is $50 \mu \mathrm{m}$. 
considered in this paper are not significant. Here the subscripts denote the model and the superscript 0 denotes pure oil and the superscript $w$ denotes pure water.

In summary then, whenever the droplet thermal conductivity exceeds the thermal conductivity of the surrounding gas and the reduced time $\tau$ exceeds 0.5 , the quantitative predictions for the reduced temperatures of the zero-gradient and finite-gradient models agree to within 10 percent and the superheat times agree to within 25 percent for pure oil droplets and to within 15 percent for pure water droplets.

The authors thank members of the Applied Mathematics Division and the Computer Sciences Division at the National Bureau of Standards for their assistance in preparing and using computer programs. They benefited from helpful discussions with T. D. Coyle, K. G. Krerker, and M. B. McNeil. One of us, Richard Kayser, Jr., gratefully acknowledges support from the National Science Foundation.

\section{Appendix A}

In this appendix, we prove that the function $\Delta$ given in eq (29) has no complex roots.

We first assume that such roots exist and then show that this assumption leads to a contradiction. Let us assume that $\rho_{1}$ and $\rho_{2}$ are nonzero and that $\Delta\left(\rho_{i}\right)=0$ where $i=1$ or 2 . We define the auxiliary functions

$$
u_{1}(\chi)=\chi^{-1} \sinh \rho_{1} \chi
$$

and

$$
u_{2}(\chi)=\chi^{-1} \operatorname{sinn}\left(\rho_{1}\right) \exp \left\{-\alpha \rho_{1}(\eta-1)\right\} .
$$

These auxiliary functions satisfy the differential equations

$$
\begin{aligned}
& \left\{\frac{d^{2}}{d \chi^{2}}+\frac{2}{\chi}-\rho_{1}^{2}\right\} u_{1}=0 \quad 0 \leq \chi<1 \\
& \left\{\frac{d^{2}}{d \chi^{2}}+\frac{2}{\chi}-\alpha^{2} \rho_{1}^{2}\right\} u_{2}=0 \quad 1<\chi
\end{aligned}
$$

and the conditions that $u_{1}(0)=0, u_{2}(\infty)=0, u_{1}(1)=u_{2}(1)$, and $u_{1}^{\prime}(1)=\beta u_{2}^{\prime}(1)$. The last condition is a consequence of the definition of $\rho_{1}$. Let $v_{1}(\chi)$ and $v_{2}(\chi)$ be the analogues of $u_{1}(\chi)$ and $u_{2}(\chi)$ with $\rho_{1}$ replaced by $\rho_{2}$ and consider eqs (A5) to (A8) to be the corresponding versions of eqs (A1) to (A4). For brevity, we do not list eqs (A5) to (A8). Equation (A3) is now multiplied by $v_{1}$, and equation (A7) is multiplied by $u_{1}$. The two are subtracted. The resulting equation is multiplied by $\chi_{1}^{2}$ and then integrated from zero to one to obtain

$$
\left(\rho_{1}^{2}-\rho_{2}^{2}\right) \int_{0}^{1} \chi^{2} u_{1} v_{1} d \chi+\int_{0}^{1} \chi^{2}\left(u_{1}^{\prime \prime} v_{1}-u_{1} \nu_{1}^{\prime \prime}\right) d \chi+\int_{0}^{1} 2 \chi\left(u_{1}^{\prime} v_{1}-u_{1} v_{1}^{\prime}\right) d \chi=0
$$

Performing the same operations on the functions $u_{2}$ and $v_{2}$ but integrating instead from one to infinity yields

$$
\alpha^{2}\left(\rho_{1}^{2}-\rho_{2}^{2}\right) \int_{1}^{\infty} \chi^{2} u_{2} v_{2} d \chi+\int_{1}^{\infty} \chi^{2}\left(u_{2}^{\prime \prime} v_{2}-u_{2} v_{2}^{\prime \prime}\right) d \chi+\int_{1}^{\infty} 2 \chi\left(u_{2}^{\prime} v_{2}-u_{2} v_{2}^{\prime}\right) d \chi=0 .
$$

Multiplying eq (A10) by $\beta$, subtracting it from eq (A9), and using the conditions on $u_{1}, v_{1}, u_{2}$, and $v_{2}$, we obtain the following condition:

$$
\left(\rho_{1}^{2}-\rho_{1}^{2}\right)\left\{\int_{0}^{1} \chi^{2} u_{1} v_{1} d \chi+\beta \alpha^{2} \int_{1}^{\infty} \chi^{2} u_{2} v_{2} d \chi\right\}=0 .
$$


We now assume that both $\rho_{1}$ and $\rho_{2}$ are complex and that $\rho_{2}=\rho_{1}^{*}$. In this case, $u_{1}^{*}=v_{1}$ and $u_{2}^{*}=v_{2}$ so that both integrals in eq (All) are of positive definite quantities and therefore necessarily positive. The curly brackets then cannot vanish implying that $\rho_{1}^{2}-\rho_{2}^{2}=0$. This criterion cannot be satisfied by $\rho_{1}$ and $\rho_{2}$ of the assumed nature. This yields the contradiction mentioned earlier and is sufficient to rule out the existence of complex roots.

\section{Appendix B}

As stated in section 5, the integrals representing the temperature distributions of the droplet and surrounding gas, $\boldsymbol{\theta}_{\boldsymbol{d}}(\boldsymbol{\eta}, \boldsymbol{\tau})$ and $\boldsymbol{\theta}_{\boldsymbol{g}}(\boldsymbol{\eta}, \boldsymbol{\tau})$ given as eqs (33) and (34), must be evaluated numerically. The same remark also holds of course for the average temperature in the droplet, $\boldsymbol{\theta}_{\boldsymbol{d}}(\mathrm{avg}, \tau)$, given as eq (35). Let us denote the denominator of the integrands of each of these three expressions by

$$
D(\xi)=\alpha^{2} \beta^{2} \xi^{2} \sin ^{2} \xi+(\xi \cos \xi-(1-\beta) \sin \xi)^{2} .
$$

When $D(\xi)$ becomes small, the integrands become large.

The values of $\alpha^{2} \beta^{2}$ for the oil and water droplets are from table 2, respectively, $1.13 \times 10^{-4}$ and $1.24 \times$ $10^{-5}$. Such small values lead to very large integrands whenever $\xi$ is near one of the minima of $D(\xi)$. We may approximate the values of $\xi_{i}$ for which $D\left(\xi_{i}\right)$ is at one of its minima by the roots of the equation

$$
\xi_{i} \cos \xi_{i}-(1-\beta) \sin \xi_{i}=0
$$

when $\alpha^{2} \beta^{2}$ is small compared to one. As a consequence of such small $\alpha^{2} \beta^{2}$, the integrands are highly oscillatory. For this reason, Gaussian-type integration formulas are inappropriate. They would require an extremely high point formula in order to obtain accurate results. The procedure used consisted of two steps which we now outline schematically. The first step is to terminate the upper limit of integration so that

$$
\int_{0}^{\infty} f(x) d x \cong \int_{0}^{R} f(x) d x,
$$

Where $R$ is choosen to make the result independent of $R$ to the desired accuracy. Secondly, a function $g(x)$ which behaves like $f(x)$ in the neighborhood of its large oscillations is introduced as follows

$$
\int_{0}^{R} f(x) d x=\int_{0}^{R}[f(x)-g(x)] d x+\int_{0}^{R} g(x) d x .
$$

The function $g(x)$ is chosen subject to two restrictions; namely, that $f(x)-g(x)$ is a relatively smoothly varying function of $x$, and secondly that the last integral in eq. (B4) may be evaluated analytically. Finally, $\int_{0}^{R}[f(x)-g(x)] d x$ is computed using a finely-spaced Simpson's rule.

We obtain a function $g(x)$ by summing three expressions derived from the first three roots, $\boldsymbol{\xi}_{1}, \boldsymbol{\xi}_{2}$, and $\boldsymbol{\xi}_{3}$ of eq (B2). The expression which corresponds to the $i$ th root is the numerator of the particular integrand in one of the eqs (33), (34), and (35) evaluated at $\xi=\xi_{i}$ with the denominator replaced by

$$
D(\xi) \rightarrow \alpha^{2} \beta^{2} \xi_{i}^{2} \sin \xi_{i}+\left(\beta \cos \xi_{i}-\xi_{i} \sin \xi_{i}\right)^{2}\left(\xi-\xi_{i}\right)^{2} .
$$

The initial conditions, $\boldsymbol{\theta}_{\boldsymbol{d}}(\boldsymbol{\eta}, 0)=0$ and $\boldsymbol{\theta}_{\boldsymbol{g}}(\eta, 0)=1$, were checked using $\mathrm{R}=20$ For $\tau \boldsymbol{\tau} \mathbf{} 0.25$, the relative error introduced in using $R=10$ instead of $R=\infty$ was never larger than $10^{-6}$ for any case. The results displayed graphically in section 6 were computed to at least four significant figures. This required varying the spacing in the Simpson's rule until the desired accuracy was achieved. The spacing was generally of the order of $10^{-4}$. 


\section{References}

[1] Chigier, N. A., in Preprints for the Symposium on Evaporation-Combustion of Fuel Droplets, presented before the Division of Petroleum Chemistry, Inc., American Chemical Society, August 29-September 3, 1976, Vol. 21, No. 4, p. 616.

[2] Williams, A., Combustion and Flame 21, 1 (1973).

[3] Dooher, J., et al., preprints, August 1976.

[4] Spadaccin, L. J., and Pelmas, R., in Preprints for the Symposium on Evaporation-Combustion of Fuel Droplets, presented before the Division of Petroleum Chemistry, Inc., American Chemical Society, August 29-September 3, 1976, Vol. 21, No. 4, p. 741.

[5] Jarvis, T. J., et al., J. of Colloid and Interface Science 50, 359 (1975).

[6] Reid, R. C., American Scientist 64, 146 (1976).

[7] Law, C. K., Combustion and Flame 26, 17 (1976).

[8] Parks, J. M., et al., AIAA Journal 4, 1632 (1966).

[9] Friedman, M. H., and Churchill, S. W., Chemical Engineering Progress Symposium Series, Heat Transfer-Boston, American Institute of Chemical Engineering 61, 1 (1965).

[10] Abramowitz, M., and Stegun, I., editors, Handbook of Mathematical Functions, (Dover Publications, Inc., New York, 1965) p. 1019.

[11] Bennett, H. S., J. Res. Nat. Bur. Stand. (U.S.), 75A (Phys. and Chem.), No. 4, 261-268 (1971). See Appendix A. In particular, we refer the reader to eqs (A1) to (A21).

[12] Ince, E. L., Ordinary Differential Equations (Dover Publications, New York, 1944). 